\title{
Genetic parameters for ostrich incubation traits in South Africa
}

\author{
Z. Brand ${ }^{1,2 \#}$, S.W.P. Cloete ${ }^{1,3}$, I.A. Malecki ${ }^{4}$ and C.R. Brown ${ }^{5}$ \\ ${ }^{1}$ Department of Animal Sciences, University of Stellenbosch, Private Bag X1, Matieland 7602, South Africa \\ ${ }^{2}$ Institute for Animal Production, Oudtshoorn, PO Box 313, Oudtshoorn 6620, South Africa \\ ${ }^{3}$ Institute for Animal Production, Elsenburg, Private Bag X1, Elsenburg 7607, South Africa \\ ${ }^{4}$ School of Biology, Faculty of Natural and Agricultural Science, University of Western Australia, Crawley, Australia \\ ${ }^{5}$ Institute of Science and the Environment, University of Worcester, Henwick Grove, Worcester, WR2 6AJ, UK
}

\begin{abstract}
Data obtained from a pair-mated ostrich flock located at Oudtshoorn, South Africa, were used to estimate genetic parameters for egg weight (EWT), weight of day-old chicks (CWT), water loss to 21 (WL21) and 35 (WL35) days of incubation, and pipping time (PT) for between 13806 and 19913 artificially incubated ostrich eggs during the $2003-2006$ production years. Data were initially analysed as single traits using ASREML. Covariance components and ratios were subsequently derived from two-trait analyses. Single-trait estimates of heritability $\left(\mathrm{h}^{2}\right)$ were $0.46 \pm 0.08$ for EWT, $0.34 \pm 0.07$ for CWT, $0.34 \pm 0.07$ for WL21, $0.27 \pm 0.06$ for WL35 and $0.16 \pm 0.04$ for pipping time. Estimates of maternal genetic effects $\left(\mathrm{m}^{2}\right)$ were $0.23 \pm 0.12$ for EWT and $0.29 \pm 0.10$ for CWT. A maternal permanent environmental effect amounted to $0.25 \pm 0.10$ for EWT, $0.12 \pm 0.09$ for CWT, $0.25 \pm 0.04$ for WL21 and $0.30 \pm 0.04$ for WL35. Genetic correlations with EWT amounted to $-0.21 \pm 0.13$ for WL21 and to $-0.12 \pm 0.14$ for WL35. Corresponding correlations with CWT were $-0.43 \pm 0.07$ and $-0.54 \pm 0.11$. Parameters indicate that it should be possible to alter evaporative water loss of ostrich eggs by genetic selection. A feasible selection strategy, however, needs to be devised as it is challenging to effect genetic change in a trait with an intermediate optimum.
\end{abstract}

Keywords: Embryo, shell, albumen, water loss, egg membranes, air sac, development, regression

\#Corresponding author. E-mail: zanellb@elsenburg.com

\section{Introduction}

Genetic make-up is one of the factors influencing the performance of individuals and genetic improvement may be achieved by selection for certain traits (Petitte \& Davis, 1999). During the past few decades extensive research has been carried out on selective breeding to improve production traits in species of common domestic livestock. Genetic and crossbreeding parameters, as well as line and breed differences for these livestock species are thus readily available. Access to this information ensures structured breeding programmes, involving line- and cross-breeding and exploiting sexual dimorphism and heterosis.

In the ostrich industry, however, little is known about either genetic parameters or responses to selection for specific traits. Definite breeding objectives and industry breeding structures are largely absent (Cloete et al., 2002; 2008), as past selection was largely based on feather characteristics. Moreover, typical ostrich production systems, such as flock-mating, communal nesting systems and a very narrow male to female ratio, also present challenges for implementing selective breeding programmes aimed at improving some specific traits (Cloete et al., 1998). Van Schalkwyk et al. (1996) and Cloete et al. (1998) were the first to report estimates for repeatability of, and phenotypic correlations among, reproductive traits in the ostrich. Subsequent estimates of genetic parameters for egg, chick and reproductive traits are still limited to only a few studies (Bunter et al., 1999; 2001; Bunter \& Cloete, 2004; Cloete et al., 2004; 2005; Lambrechts, 2004). Egg quality is also reported to have a significant genetic component (Stewart, 1995). No information is available on genetic parameters for ostrich incubation traits, although systematic factors affecting ostrich incubation traits were recently estimated by Brand et al. (2008). These results showed that evaporative water loss from artificially incubated ostrich eggs to 35 days of incubation depended on the interaction between year and season and the incubator used. The time interval from the commencement of incubation to external pipping depended on the interaction between year and season, the storage period of eggs prior to incubation, and the incubator used. These systematic effects thus need to be considered during the genetic analysis of incubation traits in ostriches. Within-season evaporative water loss has also been shown to be repeatable in 
ostrich females (Blood et al., 1998). No other reports of inter-individual variation were found for incubation traits in ostriches.

Evaporative water loss is known to be curvilinear related to embryonic mortality (Blood et al., 1998), and is therefore of importance for artificial incubation. The general lack of genetic parameters for incubation traits has previously been highlighted as a limitation to efficient commercial ostrich production (Cloete et al., 2002) and it is therefore evident that a better understanding of genetic factors influencing the hatchability of ostrich eggs is essential (Cloete et al., 2002). The aim of this study therefore was to estimate genetic parameters for egg weight, chick weight, water loss and pipping time of ostrich eggs.

\section{Material and Methods}

The study was carried out over the 2003 - 2006 breeding seasons. The experimental population used for the study was the commercial, pair-bred ostrich flock at the Oudtshoorn Research Farm in the Klein Karoo region of South Africa. The origin of the flock and the general management procedures implemented have been described previously (Van Schalkwyk et al., 1998; Bunter \& Cloete, 2004). Brand et al. (2008) recently reported the effects of a number of systematic factors on egg weight after collection (EWT), the percentage of water loss from eggs up to 21 and 35 days of incubation (respectively WL21 and WL35), as well as chick weight at one-day-old (CWT) and the interval from the commencement of incubation to external pipping (PT). The latter reference can be consulted with regard to trait definitions and the effects of the relevant systematic factors. The present investigation estimated genetic parameters for the traits considered. The same analyses used for the estimation of systematic effects were used to estimate variance components for the traits set out above. An animal model was used, which attributed each EWT, WL21, WL35, CWT and PT record to an individual female. Maternal effects of the female were modelled by fitting female permanent environmental $\left(\mathrm{c}^{2}\right)$ and maternal genetic $\left(\mathrm{m}^{2}\right)$ effects. Initial analyses also included the effect of the breeding paddock occupied by the parents producing a specific egg/chick, but this effect was not significant for the egg and incubation traits that were considered and was consequently omitted from subsequent analyses. Random terms were then added to the operational model including only fixed and interaction effects (Model 1), resulting in the following models for analyses (in matrix notation):

$$
\begin{aligned}
& y=X b+Z_{1} a+e \\
& y=X b+Z_{1} a+Z_{2} c+e \\
& y=X b+Z_{1} a+Z_{3} m+e \\
& y=X b+Z_{1} a+Z_{3} m+Z_{2} c+e
\end{aligned}
$$

(Model 5)

In these analyses, y was a vector of observations for EWT, WL21, WL35, CWT and PT records, and b, a, $\mathrm{m}$ and c vectors of fixed effects, direct genetic variances, maternal genetic variances and maternal permanent environmental variances respectively. $\mathrm{X}, \mathrm{Z}_{1}, \mathrm{Z}_{2}$ and $\mathrm{Z}_{3}$ were the corresponding incidence matrices relating the respective effects to $y$, while e was the vector of residuals.

It was assumed that:

$$
\mathrm{V}(\mathrm{a})=\mathrm{A} \sigma_{\mathrm{a}}^{2} ; \mathrm{V}(\mathrm{m})=\mathrm{A} \sigma_{\mathrm{m}}^{2} ; \mathrm{V}(\mathrm{c})=\mathrm{I} \sigma_{\mathrm{c}}^{2} ; \mathrm{V}(\mathrm{e})=\mathrm{I} \sigma_{\mathrm{e}}^{2}
$$

With A being the matrix describing relationships between animals (the numerator relationship matrix), I being identity matrices with the order corresponding to the number of dams for dam permanent environment and the number of records for the residual; $\sigma_{\mathrm{a}}{ }_{\mathrm{a}}, \sigma_{\mathrm{m}}^{2}, \sigma_{\mathrm{c}}{ }_{\mathrm{c}}$ and $\sigma_{\mathrm{e}}{ }_{\mathrm{e}}$ the direct genetic variance, maternal genetic variance and the maternal permanent environmental variance and environmental (residual) variance respectively. These analyses yielded estimates of genetic and permanent environmental variances. Ratios for direct additive genetic, maternal genetic as well as maternal permanent environmental variances were computed from these estimates. These variances were expressed relative to the total phenotypic variance.

Random terms were added to analytical models sequentially. Likelihood Ratio Tests (LRT) were used to assess the significance of the contribution of each random term to improvements in the model. The LRT is based on testing the increase in Log-likelihood resulting from adding an additional random term to the model of analysis (starting with model 1 ) as a $\mathrm{Chi}^{2}$ statistic. When two models included the same number of random terms, the model with the higher value for the Log-likelihood was preferred (i.e. when Model 3 was 
compared to Model 4). The ASREML programme was used for estimations of fixed effects and subsequently to derive variance components for each trait in single-trait analyses (Gilmour et al., 1999). ASREML estimates variance components for mixed models by residual maximum likelihood, employing an average information algorithm that concurrently provides estimates of standard errors for parameters (Gilmour et al., 1995). A series of two-trait analyses were then performed to estimate direct genetic, maternal genetic, maternal permanent environmental and environmental correlations between traits.

\section{Results and Discussion}

Log-likelihood values under alternative random effects models are presented in Table 1. The addition of the direct additive effect as a single random effects resulted in significant improvements in the LRT throughout. When further random effects were added, the LRT indicated that models for WL21, WL35 and PT should include direct additive and dam permanent environmental effects as random sources of variation. Models including direct additive, maternal additive and permanent environmental effects were used for EWT and CWT.

Table 1 Log-likelihood values for the respective traits under different random effects models, with the best model for each trait represented in bold italic figures

\begin{tabular}{lccccc}
\hline Trait & Model 1 & Model 2 & Model 3 & Model 4 & Model 5 \\
\hline EWT & 1115.21 & 1144.36 & 1568.28 & 1730.93 & $\mathbf{1 7 3 4 . 9 8}$ \\
CWT & 3984.6 & 8714.84 & 8835.6 & 8839.48 & $\mathbf{8 8 4 0 . 6 7}$ \\
WL21 & -3046.62 & -9054.17 & $\mathbf{- 9 0 2 2 . 6 9}$ & -9024.34 & -9021.74 \\
WL35 & -1650.57 & -7168.01 & $\mathbf{- 7 1 0 8 . 2 3}$ & -7128.71 & -7107.61 \\
PT & -10525.5 & 10043.9 & $\mathbf{- 1 0 0 3 8 . 8}$ & -10043.1 & -10038.8 \\
\hline
\end{tabular}

EWT = egg weight at time of lay; CWT = chick weight at one-day-old; WL21 = water loss at 21 days of incubation; WL35 = water loss at 35 days of incubation; PT = incubation time to external pipping.

Initially, variance components and ratios were estimated for the five traits (EWT, CWT, WL21, WL35 and PT) in single-trait analyses (Table 2). Estimated heritabilities $\left(h^{2}\right)$ for EWT and CWT were high at 0.46 and 0.34, respectively. These estimates were somewhat higher than those previously reported by Bunter et al. (1999) $(\mathrm{EWT}=0.21$; CWT $=0.13)$ and Bunter \& Cloete (2004) $(\mathrm{EWT}=0.19$; CWT $=0.16)$. The higher estimates of $\mathrm{h}^{2}$ could be associated with an improved data structure for the partitioning of sire, dam and paddock effects, caused by the intentional re-allocation of specific females to different mates and breeding paddocks, as recommended by Bunter (2002). Estimates of $h^{2}$ were also high for evaporative water loss at 0.34 for WL21 and 0.27 for WL35. The heritability for PT was moderate at 0.16 . No previous heritability estimates were found for the latter three traits in ostriches, but Blood et al. (1998) reported that water loss from ostrich eggs were highly repeatable within a production season. The $\mathrm{h}^{2}$ estimate for PT is somewhat lower than a comparable estimate of 0.49 for incubation time in female breeder broilers (Suarez et al., 1997). All $\mathrm{h}^{2}$ estimates were significant. The maternal genetic effect $\left(\mathrm{m}^{2}\right)$ was only significant for the egg traits and was moderate to high both for EWT and CWT at $0.19 \pm 0.11$ and $0.28 \pm 0.11$, respectively. The estimate for EWT was slightly lower than a comparable estimate of 0.31 published by Bunter \& Cloete (2004). The present estimate for CWT, however, was consistent with an estimate of 0.26 reported by Bunter \& Cloete (2004). Hen permanent environment variation $\left(\mathrm{c}^{2}\right)$ accounted for $<15 \%$ of the phenotypic variation in CWT and PT. In contrast, it contributed between 20 and 30\% of the phenotypic variation to EWT, WL21 and WL35. 
Table 2 Estimates of variance components and ratios ( \pm s.e.) for EWT, CWT, WL21, WL35 and PT from single-trait analysis

\begin{tabular}{lccccc}
\hline Effect & EWT & CWT & WL21 & WL35 & PT \\
\hline Components & & & & & \\
$\sigma_{a}^{2}$ & 0.008 & 0.003 & 1.41 & 2.65 & 0.27 \\
$\sigma_{m}^{2}$ & 0.003 & 0.003 & - & - & - \\
$\sigma_{c}^{2}$ & 0.010 & 0.001 & 1.01 & 2.89 & 0.08 \\
$\sigma_{p}^{2}$ & 0.020 & 0.010 & 4.09 & 9.80 & 1.71 \\
$\sigma_{e}^{2}$ & 0.001 & 0.003 & 1.68 & 4.26 & 1.37 \\
Ratios & & & & & \\
$h^{2}$ & $0.46 \pm 0.08$ & $0.34 \pm 0.07$ & $0.34 \pm 0.07$ & $0.27 \pm 0.06$ & $0.16 \pm 0.04$ \\
$m^{2}$ & $0.19 \pm 0.11$ & $0.28 \pm 0.11$ & - & - & - \\
$c^{2}$ & $0.28 \pm 0.10$ & $0.13 \pm 0.09$ & $0.25 \pm 0.04$ & $0.29 \pm 0.04$ & $0.04 \pm 0.01$ \\
\hline$\sigma_{e}=$ & & & & \\
\hline
\end{tabular}

$\sigma_{e}^{2}=$ environmental (residual) variance component, $\sigma_{a}^{2}=$ direct additive genetic variance, $\sigma_{m}^{2}=$ maternal additive genetic variance, $\sigma_{c}^{2}=$ dam permanent environmental variance, $\sigma_{p}^{2}=$ phenotypic variance, $h^{2}=$ direct heritability, $m^{2}=$ maternal heritability, $c^{2}=$ ratio of permanent environmental variance to phenotypic variance.

EWT - egg weight; CWT - chick weight at one-day-old; WL21 and WL35 - the percentage of water loss from eggs up to 21 and 35 days of incubation, respectively; PT - interval from the commencement of incubation to external pipping.

Estimates of genetic parameters from a series of two-trait analyses are presented in Table 3. Estimates of $h^{2}$ for EWT and CWT were slightly higher than those reported from single-trait analysis, whereas estimates for WL21 and WL35 were slightly lower. Heritability estimates for PT were consistent with those obtained from single-trait analysis. In broilers, the genetic correlation between egg weight and embryo weight is near zero at the beginning of incubation, but it increases to a maximum at hatch (Suarez et al., 1997). In the present study, egg weight and day-old chick weight were highly correlated on the genetic level $(0.97 \pm 0.01)$, which corresponds with the previous estimate of 0.95 reported by Bunter \& Cloete (2004). This high genetic correlation indicates that egg weight and chick weight are essentially the same trait, that is, they are governed by a largely similar set of genes. The strong positive correlation between egg weight and chick weight at hatch is fairly constant across species (Wilson, 1991a) with chick weight being primarily determined by initial egg weight, although it is also affected by evaporative water loss during incubation.

Genetic correlations of WL with EWT were negative, but not significantly different from zero. A negative correlation was expected due to the surface area : volume ratio, which results in heavier eggs, at least in theory, being expected to lose less water than light eggs with a wider surface area : volume ratio. It is conceded that this relationship is not a simple one as other factors such as eggshell structure and porosity also contribute to water loss. Direct genetic correlations of WL with CWT were negative and high at -0.73 and -0.54 , respectively, for WL21 and WL35. This result was expected since eggs with high levels of water loss will produce lighter chicks as a result of dehydration. This principle is well illustrated in small domestic poultry species, as demonstrated by Tullett \& Burton (1982) and Davis et al. (1988). The direct genetic correlation between WL21 and WL35 amounted to $1.00 \pm 0.0003$, suggesting that these traits are essentially the same. Pipping time has no significant genetic correlation with any of the other traits with estimated correlations ranging from $-0.04 \pm 0.17$ with WL35 to $0.22 \pm 0.15$ with EWT. This result corresponds with findings by Suarez et al. (1997) that incubation time and egg weight of broiler breeders were not highly correlated. The maternal additive correlation was only significant for EWT and CWT with a very high correlation of 0.99. The corresponding correlation reported by Bunter \& Cloete (2004) amounted to 0.96.

The hen permanent environmental correlation between EWT and CWT was high at $0.97 \pm 0.35$. This value is slightly higher than the value of 0.89 reported by Bunter \& Cloete (2004). Hen permanent environmental correlations of EWT with WL were low and not significant. The corresponding maternal permanent environmental correlations of CWT with WL were negative and in the order of -0.50 in magnitude. On the maternal permanent environmental level, EWT was positively related to PT, the estimate 
approaching 0.60 (Table 3). This result seems to imply that larger eggs are likely to hatch later on the dam permanent environmental level. Dam permanent environmental correlations of PT with other traits were not significant and ranged from $0.14 \pm 0.16$ with WL21 to $0.45 \pm 0.27$ for CWT.

Table 3 Estimates of heritability $\left(\mathrm{h}^{2}\right)$, maternal genetic effects $\left(\mathrm{m}^{2}\right)$, maternal permanent environmental effects $\left(c^{2}\right)$, and correlations between these effects, residual and phenotypic variance and correlations between egg weight (EWT), chick weight (CWT), water loss to 21 days (WL21), water loss to 35 days (WL35) and pipping time (PT) from multi-trait analyses

\begin{tabular}{|c|c|c|c|c|c|}
\hline Traits & EWT & CWT & WL21 & WL35 & PT \\
\hline \multicolumn{6}{|c|}{ Additive genetic correlations ( $\mathrm{h}^{2}$ in bold) } \\
\hline $\begin{array}{l}\text { EWT } \\
\text { CWT } \\
\text { WL21 } \\
\text { WL35 } \\
\text { PT }\end{array}$ & $0.49 \pm 0.08$ & $\begin{array}{l}0.97 \pm 0.01 \\
\mathbf{0 . 3 7} \pm \mathbf{0 . 0 7}\end{array}$ & $\begin{array}{c}-0.21 \pm 0.13 \\
-0.73 \pm 0.07 \\
\mathbf{0 . 3 4} \pm \mathbf{0 . 0 7}\end{array}$ & $\begin{array}{c}-0.12 \pm 0.14 \\
-0.54 \pm 0.11 \\
1.00 \pm 0.00 \\
\mathbf{0 . 2 6} \pm \mathbf{0 . 0 6}\end{array}$ & $\begin{array}{r}0.22 \pm 0.15 \\
0.12 \pm 0.16 \\
0.07 \pm 0.17 \\
-0.04 \pm 0.17 \\
\mathbf{0 . 1 6} \pm \mathbf{0 . 0 4}\end{array}$ \\
\hline \multicolumn{6}{|c|}{ Maternal genetic correlations ( $\mathrm{m}^{2}$ in bold) } \\
\hline $\begin{array}{l}\text { EWT } \\
\text { CWT } \\
\text { WL21 } \\
\text { WL35 } \\
\text { PT }\end{array}$ & $0.23 \pm 0.12$ & $\begin{array}{l}0.99 \pm 0.03 \\
\mathbf{0 . 2 9} \pm \mathbf{0 . 1 0}\end{array}$ & $\begin{array}{l}- \\
- \\
-\end{array}$ & $\begin{array}{l}- \\
- \\
- \\
-\end{array}$ & $\begin{array}{l}- \\
- \\
- \\
- \\
-\end{array}$ \\
\hline \multicolumn{6}{|c|}{ Permanent environmental correlations ( $\mathrm{c}^{2}$ in bold) } \\
\hline $\begin{array}{l}\text { EWT } \\
\text { CWT } \\
\text { WL21 } \\
\text { WL35 } \\
\text { PT }\end{array}$ & $0.25 \pm 0.10$ & $\begin{array}{l}0.97 \pm 0.35 \\
\mathbf{0 . 1 1} \pm \mathbf{0 . 0 9}\end{array}$ & $\begin{array}{c}0.11 \pm 0.14 \\
-0.47 \pm 0.12 \\
\mathbf{0 . 2 5} \pm \mathbf{0 . 0 4}\end{array}$ & $\begin{array}{c}0.02 \pm 0.13 \\
-0.54 \pm 0.11 \\
1.00 \pm 0.00 \\
\mathbf{0 . 3 0} \pm \mathbf{0 . 0 4}\end{array}$ & $\begin{array}{c}0.60 \pm 0.17 \\
0.45 \pm 0.27 \\
0.14 \pm 0.16 \\
0.155 \pm 0.15 \\
\mathbf{0 . 0 4} \pm \mathbf{0 . 0 1}\end{array}$ \\
\hline \multicolumn{6}{|c|}{ Residual correlation ( $\sigma_{\mathrm{e}}^{2}$ in bold $)$} \\
\hline $\begin{array}{l}\text { EWT } \\
\text { CWT } \\
\text { WL21 } \\
\text { WL35 } \\
\text { PT }\end{array}$ & 0.0008 & $\begin{array}{c}0.31 \pm 0.03 \\
\mathbf{0 . 0 0 2}\end{array}$ & $\begin{array}{c}-0.01 \pm 0.21 \\
-0.33 \pm 0.11 \\
\mathbf{1 . 6 8}\end{array}$ & $\begin{array}{c}-0.19 \pm 0.21 \\
-0.53 \pm 0.06 \\
0.86 \pm 0.01 \\
\mathbf{4 . 3 0}\end{array}$ & $\begin{array}{c}-0.07 \pm 0.12 \\
-0.16 \pm 0.05 \\
0.04 \pm 0.04 \\
0.07 \pm 0.03 \\
\mathbf{1 . 3 7}\end{array}$ \\
\hline \multicolumn{6}{|c|}{ Phenotypical correlation $\left(\sigma_{\mathrm{p}}^{2}\right.$ in bold) } \\
\hline $\begin{array}{l}\text { EWT } \\
\text { CWT } \\
\text { WL21 } \\
\text { WL35 } \\
\text { PT }\end{array}$ & 0.02 & $\begin{array}{c}0.86 \pm 0.01 \\
\mathbf{0 . 0 1}\end{array}$ & $\begin{array}{c}-0.06 \pm 0.04 \\
-0.51 \pm 0.03 \\
\mathbf{4 . 1 2}\end{array}$ & $\begin{array}{c}-0.06 \pm 0.04 \\
-0.48 \pm 0.03 \\
0.95 \pm 0.002 \\
\mathbf{9 . 8 1}\end{array}$ & $\begin{array}{c}0.11 \pm 0.02 \\
-0.01 \pm 0.02 \\
0.05 \pm 0.02 \\
0.05 \pm 0.02 \\
\mathbf{1 . 7 1}\end{array}$ \\
\hline
\end{tabular}

The residual correlation between EWT and CWT was substantially lower than unity at $0.31 \pm 0.03$. The corresponding correlation reported by Bunter \& Cloete (2004) amounted to 0.57. The estimated phenotypic variances for the respective traits were largely similar to the estimates from single-trait analyses. There was also a strong phenotypic correlation $\left(r_{p}\right)$ between egg and chick weight $(0.86 \pm 0.01)$, while the phenotypic correlations between EWT and WL21, Wl35 and PT were low and not significant. 


\section{Conclusion}

Estimates of $\mathrm{h}^{2}$ indicate that it is possible to alter evaporative water loss of ostrich eggs by genetic selection. The only previous reference suggesting that selection for water loss may result in current flock gains was the finding of Blood et al. (1998) that the within-season water loss of eggs from individual females was highly repeatable. A feasible selection strategy, however, needs to be devised, as it may be challenging to effect genetic change in a trait with an intermediate optimum. Egg and day-old chick weights, as well as pipping time, were also demonstrated to be heritable, and should respond to selection, should change be desired. The potential role of these traits in an integrated breeding scheme, however, needs further study before being applied in the industry. It is conceded that the standardisation of egg weight to reduce the variation in evaporative water loss during artificial incubation may assist in alleviating the present high level of embryonic mortalities. However, larger chicks are generally favoured by commercial chick raisers. Grade 1 chicks in South Africa are expected to be heavier than 750 g (Verwoerd et al., 1999), exerting a direct influence on the price realised for day-old chicks. If it is considered that day-old chick weight should be about $60 \%$ of egg weight (Wilson, 1991b), this implies that eggs to be set should be at least $1250 \mathrm{~g}$ in weight. The lower 95\% confidence limit for eggs used in the present study was $1156 \mathrm{~g}$, indicating that a substantial number of the eggs used were below this recommended weight. An additional advantage of producing heavier day-old chicks is an improved chick survival to one month of age (Cloete et al., 2001), provided that the chicks do not suffer from other complications such as oedema owing to insufficient water loss.

Direct and maternal genetic correlations among traits were either high to very high in the desired direction, or small in magnitude. Given the high levels of incubation failure, further research is urgently needed to gain a better understanding of the mechanisms involved and strategies with the potential to improve incubation success.

\section{Acknowledgements}

The authors thank the South African Department of Trade and Industry for partial funding of the work through their THRIP programme, as well as all those involved in the maintenance and recording of the research flock at the Oudtshoorn Research farm.

\section{References}

Brand, Z., Cloete, S.W.P., Brown, C.R. \& Malecki, I.A., 2008. Systematic factors that affect ostrich incubation traits. S. Afr. J. Anim. Sci. 38, 315-325.

Blood, J.R., Van Schalkwyk, S.J., Cloete, S.W.P. \& Brand, Z., 1998. Embryonic deaths in relation to water loss of artificially incubated ostrich eggs. Proc. $2^{\text {nd }}$ Int. Ratite Congress, Oudtshoorn, South Africa. pp. 148-151.

Bunter, K.L., 2002. The genetic analysis of reproduction and production traits recorded for farmed ostriches (Struthio camelus). PhD thesis, University of New England, Australia.

Bunter, K.L. \& Cloete, S.W.P., 2004. Genetic parameters for egg-, chick- and live-weight traits recorded in farmed ostriches (Struthio camelus). Livest. Prod. Sci. 91, 9-22.

Bunter, K.L., Cloete, S.W.P. \& Van Schalkwyk, S.J., 1999. Significant genetic parameters for egg, chick and juvenile weight traits in ostriches. Proc. Assoc. Advmt. Anim. Breed Genet. 13, 476-479.

Bunter, K.L., Cloete, S.W.P., Van Schalkwyk, S.J. \& Graser, H.U., 2001. Factors affecting reproductive performance in farmed ostriches. Proc. Assoc. Advmt. Anim. Breed Genet. 16, 43-45.

Cloete, S.W.P., Bunter, K.L. \& Brand, Z., 2005. Phenotypical performance of ostrich females selected for live weight or chick production relative to an unselected control line. Proc. ${ }^{\text {rd }}$ Int. Ratite Sci. Sym. Madrid, Spain. pp. 99-104.

Cloete, S.W.P., Bunter, K.L., Brand, Z. \& Lambrechts, H., 2004. Co-variances for reproduction, egg weight and chick weight in ostriches. S. Afr. J. Anim. Sci. 34, 17-19.

Cloete, S.W.P., Bunter, K.L. \& Van Schalkwyk, S.J., 2002. Progress towards a scientific breeding strategy for ostriches. Proc. $7^{\text {th }}$ Wrld Cong. Gen. Appl. Livest. Prod. 30, 561-568. 18-23 August, Montpellier, France.

Cloete, S.W.P., Engelbrecht, A., Olivier, J.J. \& Bunter, K.L., 2008. Deriving a preliminary breeding objective for commercial ostriches. Aust. J. Exp. Agric. 48, 1247-1256. 
Cloete, S.W.P., Lambrecht, H., Punt, K. \& Brand, Z., 2001. Factors related to high levels of ostrich chick mortality from hatching to 90 days of age in an intensive rearing system. J. S. Afr. Vet. Assoc. 72, 197-202.

Cloete, S.W.P., Van Schalkwyk, S.J. \& Brand, Z., 1998. Ostrich breeding - progress towards a scientifically based strategy. Proc. $2^{\text {nd }}$ Int. Ratite Cong, Oudtshoorn, South Africa. pp. 55-62.

Davis, T.A., Shen, S.S. \& Ackerman, R.A., 1988. Embryonic osmoregulation: consequences of high and low water loss during incubation of the chicken egg. J. Exp. Zool. 245, 144-156.

Gilmour, A.R., Cullis, B.R., Welham, S.J. \& Thompson, R., 1999. ASREML - Reference Manual, NSW Agriculture Biometric Bulletin No. 3 NSW Agriculture, Orange Agriculture Institute, Forest Road, Orange 2800, NSW, Australia.

Gilmour, A.R., Thompson, R. \& Cullis, B.R., 1995. Average information REML, an efficient algorithm for variance parameter estimation in linear mixed models. Biometrics 51, 1440-1450.

Lambrechts, H., 2004. Reproductive efficiency of ostriches (Struthio camelus). PhD. dissertation, University of the Free State, Bloemfontein, South Africa.

Petitte, J.N. \& Davis, G., 1999. Breeding and Genetics. In: The Ostrich: Biology, Production and Health. Ed. Deeming, D.C., CABI Publishing, CAB International, Wallingford. pp. 275-292.

Stewart, J.S., 1995. Hatchery management in ostrich production. American Ostrich, November 1995. pp. 22-44.

Suarez, M.E., Wilson, H.R., Mather, F.B., Wilcox, C.J. \& McPherson, B.E., 1997. Effects of strain and age of the broiler breeder female on incubation time and chick weight. Poult. Sci. 76, 1029-1036.

Tullett, S.G. \& Burton, F.G., 1982. Factors affecting the weight and water status of the chick at hatch. Br. Poult. Sci. 23, 361-369.

Van Schalkwyk, S.J., Brand, Z., Cloete, S.W.P. \& Blood, J.R., 1998. The influence of different disinfection protocols on the hatching performance of ostrich eggs. Proc. $2^{\text {nd }}$ Int. Ratite Cong, Oudtshoorn, South Africa. pp. 157-159.

Van Schalkwyk, S.J., Cloete, S.W.P. \& De Kock, J.A., 1996. Repeatability and phenotypic correlations for live weight and reproduction in commercial ostrich breeding pairs. Br. Poult. Sci. 37, 953-962.

Verwoerd, D.J., Deeming, D.C., Angel, C.R. \& Perelman, B., 1999. Rearing environments around the world. In: The Ostrich: Biology, Production and Health. Ed. Deeming, D.C., CABI Publishing, CAB International, Wallingford. pp. 190-216.

Wilson, H.R., 1991a. Effect of egg size on hatchability, chick size and post-hatching growth. In: Avian Incubation. Ed. Tullet, S.G., Butterworth-Heinemann Ltd. Surrey, UK. pp. 279-283.

Wilson, H.R., 1991b. Interrelationship of egg size, chick size, posthatching growth and hatchability. Wrld Poult. Sci. J. 47, 5-20. 